\title{
Numerical Simulation of Initial Microstructure Evolution of Fe-C Alloys Using a Phase-field Model
}

\author{
Machiko ODE and Toshio SUZUKI \\ Department of Metallurgy, School of Engineering, The University of Tokyo, Hongo, Bunkyo-ku, Tokyo 113-8656 Japan. \\ (Received on October 16, 2001; accepted in final form on January 18, 2002)
}

\begin{abstract}
Microstructure evolution during the rapid solidification of $\mathrm{Fe}-\mathrm{C}$ and $\mathrm{Fe}-\mathrm{C}-\mathrm{P}$ alloys is simulated using the phase-field model for alloys with thin interface limit parameters. Heat transfer equation is solved simultaneously to study the heat flow and the effect of latent heat generation on the microstructure. The calculations have been carried out using a double grid method and parallel computing technique. The competitive growth of growing cells is reproduced, and the cellular/dendritic transition is also observed. Since there is a negative thermal gradient in front of a leading tip, the growth can be regarded as unidirectional free dendrite growth. The microstructure changes depending on the preferred growth orientation and impurity are also studied. The secondary arms grow preferably towards inside of the melt and develop well with increase of the tilted angle. The secondary and primary arm spacing decrease by the small amount of phosphorus addition. The time change of averaged surface temperature depending on the initial undercooling shows that the surface undercooling is always observed even when the initial value is zero.
\end{abstract}

KEY WORDS: Fe-C alloy; rapid solidification; micro-structure; dendrite; phase-field model.

\section{Introduction}

In the near-net-shape casting, such as strip casting, the surface quality may limit the applications. To understand the microstructure evolution in the vicinity of cast surface, some experimental and theoretical studies has been done in the last decade. ${ }^{1-6)}$ In experimental work, the direct measurement of surface temperature and the observation of solidification structure have been done to understand the relationship between microstructure and the cooling conditions. The melt surface is undercooled largely and rapidly after the touch to the chill plate and is cooled again after a recalescense. ${ }^{6}$ ) The double recalescense is observed when the primary phase is metastable and changes into stable one. ${ }^{3)}$ It is also observed that the solidification structure starts with a cellular structure and then changes into dendritic one within a few hundreds micrometers from the surface. ${ }^{6)}$ The primary and secondary dendrite arm spacings, grain size, and the phase transformation depending on the solidification condition are also measured.

The one-dimensional heat transfer analysis has done in the theoretical works. By fitting heat transfer calculation to the cooling rate estimated from the surface temperature of the melt by a direct measurement, ${ }^{1,4,6,7)}$ the heat transfer coefficient and solidification front temperature are estimated. So far, there is no theoretical study about a microstructure evolution because it is difficult to consider heat transfer and solute diffusion with non-planner interface at the same time. It will be important to predict microstructure patterns, such as micro segregation, as the strip casting technique comes into practical use.
A phase-field model is expected as a powerful tool for describing the complex microstructure evolution during solidification since the model satisfies the local equilibrium condition at low interface velocity and reproduces the solute trapping phenomena at high velocity regardless of the interface shape. The model was originally proposed for simulating the dendrite growth from an undercooled pure melt and has been extended to solidification of alloys. ${ }^{8-14)}$ By adopting the thin interface limit to derive the phase-field mobility, the applicability of the phase-field model is considerably widened. ${ }^{15,16)}$

Since the thermal diffusivity is much larger than the solutal one in metal system, for example three hundred times difference in $\mathrm{Fe}-\mathrm{C}$ alloy system, the effect of heat transfer has been regards as negligibly small comparing to that of solute in the existing phase-field calculations for alloys. The thermal effect should be, however, considered in the early stage of rapid solidification. When there is a large difference between diffusivity in governing equations, the double grid method is very useful to save the calculation time. The method enables us to use a large time step and save memory space at the same time. In this study, the initial microstructure evolution of $\mathrm{Fe}-0.5 \mathrm{~mol} \% \mathrm{C}$ and $\mathrm{Fe}-$ $0.5 \mathrm{~mol} \% \mathrm{C}-0.02 \mathrm{~mol} \% \mathrm{P}$ alloys during rapid solidification is reproduced using an alloy phase-filed model with the thin interface limit parameter. The thermal field equation is solved simultaneously using a double grid method and parallel computing technique to save the computational time. 


\section{Governing Equations}

\subsection{Governing Equations using Dilute Solution Ap- proximation}

The phase-field equation with $k$-hold interface energy anisotropy and the solute diffusion equation for a dilute alloy are given by, ${ }^{14,16)}$

$$
\begin{aligned}
& \frac{1}{M} \frac{\partial \phi}{\partial t}=\varepsilon^{2} \nabla^{2} \phi+\varepsilon \varepsilon^{\prime}\left[\sin (2 \theta)\left(\phi_{y y}-\phi_{x x}\right)+2 \cos (2 \theta) \phi_{x y}\right] \\
& -\frac{1}{2}\left(\varepsilon^{\prime 2}+\varepsilon \varepsilon^{\prime \prime}\right) \times\left[2 \sin (2 \theta) \phi_{x y}-\nabla^{2} \phi-\cos (2 \theta)\left(\phi_{y y}-\phi_{x x}\right)\right] \\
& +\frac{R T}{V_{\mathrm{m}}} h^{\prime}(\phi) \ln \frac{\left(1-c_{\mathrm{S}}^{\mathrm{e}}\right)\left(1-c_{\mathrm{L}}\right)}{\left(1-c_{\mathrm{L}}^{\mathrm{e}}\right)\left(1-c_{\mathrm{S}}\right)}-W g^{\prime}(\phi) \\
& \varepsilon(\theta)=\varepsilon\{1+v \cos (k \theta)\} \\
& \tan \theta=\frac{\phi_{y}}{\phi_{x}} \\
& \frac{\partial c}{\partial t}=\nabla \cdot\left[D ( \phi ) \left\{(1-h(\phi)) \cdot c_{\mathrm{L}}\left(1-c_{\mathrm{L}}\right)\right.\right. \\
& \left.\left.+h(\phi) \cdot c_{\mathrm{S}}\left(1-c_{\mathrm{S}}\right)\right\} \nabla \ln \frac{c_{\mathrm{L}}}{1-c_{\mathrm{L}}}\right] \\
& c=h(\phi) c_{\mathrm{S}}+(1-h(\phi)) c_{\mathrm{L}} \\
& \mu^{\mathrm{S}}\left(c_{\mathrm{S}}(x, t)\right)=\mu^{\mathrm{L}}\left(c_{\mathrm{L}}(x, t)\right)
\end{aligned}
$$

where $h(\phi)=\phi^{3}\left(15-10 \phi-6 \phi^{2}\right), g(\phi)=\phi^{2}(1-\phi)^{2}, \theta$ is the angle between normal direction of interface and the $x$-axis, $v$ is the magnitude of anisotropy, the superscript of $e$ represent equilibrium state and subscripts of $x x, y y$ and $x y$ represent partial derivative by $x$ or $y$ twice, and $\mathrm{S}$ and $\mathrm{L}$ represents solid and liquid phases, respectively, $R$ is gas constant, $V_{\mathrm{m}}$ is molar volume, $D(\phi)$ is solute diffusion coefficient and $W, \varepsilon$ and $M$ are phase-field parameters which are defined later. The prime of $\varepsilon$ represents the differential by $\theta$. When the preferred growth orientation is tilted from the $x$-axis with $\theta_{0}$, Eq. (2) is modified as follows.

$$
\varepsilon(\theta)=\varepsilon\left\{1+v \cos \left(k\left(\theta-\theta_{0}\right)\right)\right\}
$$

Note that the terms of $\sin (2 \theta)$ and $\cos (2 \theta)$ in Eq. (1) are substituted for the function of the partial derivative of the phase-field and they are independent of tilted angle of preferred growth direction. For a dilute ternary alloy, the governing equations and parameters are modified as in the literature. ${ }^{17)}$ In this study, temperature field is calculated simultaneously using a double grid method, in which the grid size for temperature field is set to be larger than that for phase-field and concentration field, in order to save the calculation time. The latent heat generation is estimated by summing up the change of phase-field within the corresponding temperature grid and the thermal diffusion equation is obtained as follows.

$$
\frac{\partial T}{\partial t}=\alpha \nabla^{2} T+\frac{L}{c_{p}} \sum A \cdot h^{\prime}(\phi) \frac{\partial \phi}{\partial t}
$$

where $\alpha$ is thermal diffusivity, $L$ is latent heat, $c_{p}$ is specific heat and $A$ represents the area ratio of phase-field grid to thermal one.

The phase-field parameters of $\varepsilon$ and $W$ are related to interface energy, $\sigma$, and interface width, $2 \lambda$, where $\phi$ changes from 0.1 to 0.9 , and the parameter, $M$, is related to the kinetic coefficient, $\beta .^{15,16)}$ With a dilute solution approximation, they are given by,

$$
\begin{aligned}
& \sigma=\frac{\varepsilon \sqrt{W}}{3 \sqrt{2}} \\
& 2 \lambda=2.2 \sqrt{2} \frac{\varepsilon}{\sqrt{W}} \\
& \beta=\frac{V_{\mathrm{m}}}{R T} \frac{m^{\mathrm{e}}}{1-k^{\mathrm{e}}}\left(\frac{\sigma}{M \varepsilon^{2}}-\frac{\varepsilon}{D_{\mathrm{i}} \sqrt{2 W}} \varsigma\left(c_{\mathrm{S}}^{\mathrm{e}}, c_{\mathrm{L}}^{\mathrm{e}}\right)\right) \ldots \\
& \varsigma\left(c_{\mathrm{S}}^{\mathrm{e}}, c_{\mathrm{L}}^{\mathrm{e}}\right)=\frac{R T}{V_{\mathrm{m}}}\left(c_{\mathrm{L}}^{\mathrm{e}}-c_{\mathrm{S}}^{\mathrm{e}}\right)^{2} \\
& \times \int_{0}^{1} \frac{h(\phi)[1-h(\phi)]}{[1-h(\phi)] c_{\mathrm{L}}^{\mathrm{e}}\left(1-c_{\mathrm{L}}^{\mathrm{e}}\right)+h(\phi) c_{\mathrm{S}}^{\mathrm{e}}\left(1-c_{\mathrm{L}}^{\mathrm{e}}\right)} \frac{d \phi}{\phi(1-\phi)}
\end{aligned}
$$

where $m^{\mathrm{e}}$ is equilibrium slope of liquidus, $k^{\mathrm{e}}$ is equilibrium partition coefficient and $D_{\mathrm{i}}$ is diffusion coefficient in liquid. $^{16)}$

\section{Calculations}

In the calculation, Eqs. (1), (4), (6) and (8) are used. The equations are discretized on uniform grids using an explicit finite difference scheme. The $\mathrm{Fe}-0.5 \mathrm{~mol} \% \mathrm{C}$ and $\mathrm{Fe}-$ $0.5 \mathrm{~mol} \% \mathrm{C}-0.02 \mathrm{~mol} \% \mathrm{P}$ alloys are selected as sample alloys. The physical properties for $\mathrm{Fe}-\mathrm{C}$ alloy used in calculation are shown in Table 1. The thermodynamics data for a ternary alloy is obtained from those of binary alloys under a dilute solution approximation. ${ }^{17)}$ The tilted angles between the preferred growth orientation and the $x$-axis are set to be $0,15,30$ and 45 degrees. The vanishing kinetic coefficient was used to determine the phase-field mobility. During the calculation, a stochastic noise is imposed on the liquid composition in the vicinity of interface in order to generate the fluctuations of the interface, which gives the well-developed secondary arms. The stochastic noise is set to vary composition within $1.0 \%$ of the composition where $0.01<\phi<0.5$ and the total solute content in the corresponding area is conserved.

Since the thermal diffusivity of $\mathrm{Fe}-\mathrm{C}$ alloy is about sev-

Table 1. Physical properties of $\mathrm{Fe}-0.5 \mathrm{~mol} \% \mathrm{C}$ alloy.

\begin{tabular}{l|c}
\hline \multicolumn{1}{c|}{ Properties } & Fe-C \\
\hline interface energy $\sigma\left(\mathrm{J} / \mathrm{m}^{2}\right)$ & 0.204 \\
melting point of solvent $T_{m}(\mathrm{~K})$ & 1810 \\
solute diffusivity (liquid ) $D_{L}\left(\mathrm{~m}^{2} / \mathrm{s}\right)$ & $2.0 \times 10^{-8}$ \\
solute diffusivity ( solid ) $D_{S}\left(\mathrm{~m}^{2} / \mathrm{s}\right)$ & $6.0 \times 10^{-9}$ \\
molar volume $V_{m}\left(\mathrm{~m}^{3} / \mathrm{mol}\right)$ & $7.7 \times 10^{-6}$ \\
equilibrium distribution coefficient $k_{e}$ & 0.204 \\
slope of liquids $m_{e}($ at $0.42 \mathrm{~mol} \%)(\mathrm{K} / \mathrm{mol} \%)$ & -18.02 \\
thermal diffusivity (pure iron) $\left(\mathrm{m}^{2} / \mathrm{s}\right)$ & $6.1 \times 10^{-6}$ \\
\hline
\end{tabular}




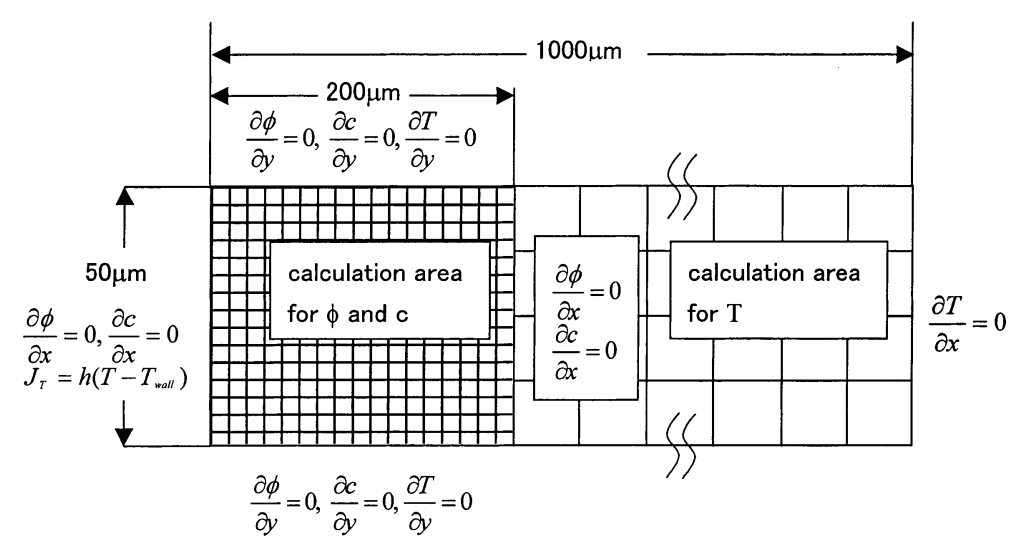

Fig. 1. Calculation area and boundary conditions for phase-field, concentration field and thermal field calculation. The grid size for phase-field and solute field, and temperature field are $0.5 \times 10^{-7} \mathrm{~m}$ and $0.5 \times 10^{-6} \mathrm{~m}$, respectively.

eral hundred times larger than the solutal one, the calculation time step is restricted by thermal field calculation. Hence, the grid size for temperature calculation is taken to be ten times larger than that for phase-field and concentration-field calculation. Before the systematic calculations, the preliminary results by the double grid method have been examined by comparing them with those by single grid method in one-dimensional and two-dimensional calculations without stochastic noise. The interface velocity and the concentration profile in one-dimensional calculations are not changed by the grid size for temperature field, and the tip radius and velocity without secondary arms in two-dimensional calculation are not affected, either. Though further examinations have not been done because of the difficulty to give the same stochastic noise effect on the interface, the small change of the microstructure, such as secondary arm spacing, can not be regard as critical problem in the double gird method considering the great improvement in computational efficiency.

The rectangular calculation area of $960 \times 4000$ grids for the phase-field and solute field and that of $96 \times 2000$ grids for the thermal field are prepared as shown in Fig. 1. A small triangle solid of $10 \times 10$ grids is initially put in the corner. The grid sizes for phase-field and concentration field, and thermal calculations are $0.5 \times 10^{-7} \mathrm{~m}$ and $0.5 \times 10^{-6} \mathrm{~m}$, respectively, and time step is $0.82 \times 10^{-5} \mathrm{~s}$. Since the grid size can be increased according to the increase of the characteristic length of the microstructure, the grid size is doubled at the middle stage of the calculation. The system is assumed to be in contact with a chill plate of a constant temperature and cooled by Newtonian cooling. The chill plate temperature and the heat transfer coefficient are set to be $600 \mathrm{~K}$ and $10000 \mathrm{~W} / \mathrm{Km}^{2}$, respectively. Since the melt surface is reported to be undercooled before nucleation in the rapid solidification processes, the phase-field calculation has been started at every $5 \mathrm{~K}$ between 0 to $25 \mathrm{~K}$ of undercooling.

The calculation has been done using a parallel computing technique in which the calculation area is divided into four blocks along the $y$-axis. A four-node parallel computer (Dec-Alpha264 $700 \mathrm{MHz}$ CPU) with IBM MPI library are used for the calculation and the typical calculation time is $72 \mathrm{~h}$ for one calculation example.

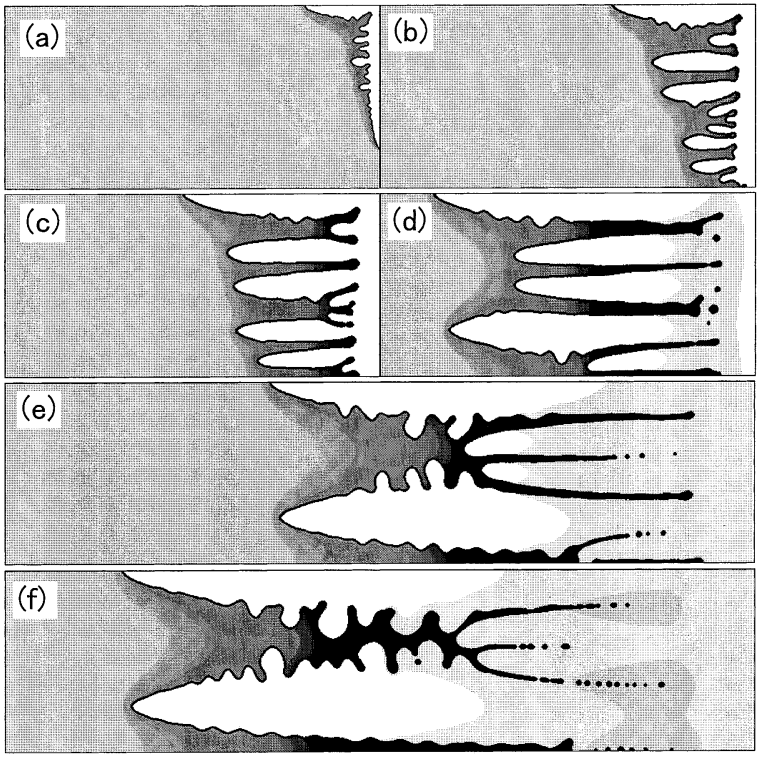

Fig. 2. The initial microstructure evolution of $\mathrm{Fe}-0.5 \mathrm{~mol} \% \mathrm{C}$ alloy. ( $\Delta T=15 \mathrm{~K}$ ) (a) $1.0 \mathrm{~ms}$, (b) $3.0 \mathrm{~ms}$, (c) $5.0 \mathrm{~ms}$, (d) $10 \mathrm{~ms}$, (e) $17 \mathrm{~ms}$, (f) $24 \mathrm{~ms}$. The secondary arm spacing at (f) changes from 10 to $5 \mu \mathrm{m}$ depending on the position.

\section{Results and Discussion}

\subsection{Microstructure Evolution during Rapid Solidifica- tion}

Figure 2 shows the microstructure evolution for $\mathrm{Fe}-$ $0.5 \mathrm{~mol} \% \mathrm{C}$ alloy during the initial stage of solidification in the case the tilted angle of preferred growth orientation is 0 degree. The initial triangle solid grows preferably into two directions. The one is growing toward inside of the melt and the other along the surface. The growth rate of the latter is larger than that of the former because the melt surface is continuously cooled. Behind the tips of both arms, the interface becomes unstable and small side branches are generated (Fig. 2(a)). Then, the side branches develop to cells and begin to grow competitively. Some of them keep growing and others stop (Fig. 2(b)). The cellular dendrite structure is observed after the competitive growth as often observed in the experiments (Fig. 2(c)). After the selection of cellular dendrite arms, the interface of advanced arms becomes unstable, developing the secondary arms (Fig. 2(d)). The other arms stop advancing and the transition from cell 
to dendrite completes. (Fig. 2(e)) At the late stage of the microstructure evolution, the secondary arms begin to coarsen (Fig. 2(f)). The fine columnar dendrite growing from the surface is shown in literature ${ }^{18)}$ for $\mathrm{Fe}-0.5$ and $2.0 \mathrm{wt} \% \mathrm{C}$ steel. Though it is observed in stainless steel but the same transition from cellular to dendritic structure shown as the typical microstructure obtained by the strip casting. ${ }^{6}$

The detailed estimation of dendrite arm spacing has been done in the analysis using a wider calculation area, in which the periodic boundary conditions are applied along the $y$-axis. Two initial solid seeds were put at the melt surface and the distance between two seeds was set to be $50 \mu \mathrm{m}$ to represent the grain size of the surface. ${ }^{1,6)}$ Other calculation conditions were the same as the cases above. The primary arm spacing $\lambda_{1}$ is $25 \mu \mathrm{m}$ and secondary arm spacing $\lambda_{2}$ changes from 10 to $5 \mu \mathrm{m}$ depending on the distance from the surface at the same calculation time as Fig. 2(f). In the experimental data, a strip cast $0.05 \mathrm{wt} \%$ carbon aluminum-killed steel has primary arm spacing of $40 \mu \mathrm{m}$ and an average secondary arm spacing of $15 \mu \mathrm{m}$. ${ }^{18)}$ For a low carbon steel with $0.038 \mathrm{wt} \% \mathrm{C}$, the primary and secondary arm spacings are 17 to $25 \mu \mathrm{m}$ and $10 \mu \mathrm{m}$, respectively. ${ }^{19)}$ Though the primary arm spacing may be slightly affected by the spacing between initial solid, the calculated arm spacings are in good agreement with the experimental data.

\subsection{Position of Leading Tip and Temperature Profile along the Growth Direction}

The tip positions depending on time are shown in Fig. 3. The position of the leading tip (arm 1 in the figure) advances rapidly from just after solidification start to about $1.0 \mathrm{~ms}$ and then the growth rate decreases considerably because the initial undercooling is decreased by the latent heat generation. Then, the tip velocity, $V$, becomes invariant and is about $6.0 \mathrm{~mm} / \mathrm{s}$. The tip velocity of the another survived arm 4 is higher than that of arm 1 in early stage but, the velocities of both tips became equal after the tip advanced to the almost the same position as arm 1 . The growth behavior of reduced arms 2, 3 and 5, are similar to each other. They advance to some position while tip velocities are reduced gradually, and are confined by the leading arms as a result of the competitive growth.

By using the values of observed growth rates, the solute diffusion length and thermal length are estimated as 2 to $3 \mu \mathrm{m}$ and 600 to $1000 \mu \mathrm{m}$, respectively. Since the arm spacing is less than $10 \mu \mathrm{m}$ in the early stage, the competitive growth of arms is dominated by solute diffusion. During the growth, the primary arm spacing increases gradually up to $25 \mu \mathrm{m}$. Since the arm spacing becomes large comparing to the diffusion length, the thermal diffusion may affect on tip growth after the primary arm spacing approaches to a constant value.

Figure 4 shows the temperature profile along the growth direction of the leading tip (arm 1). All curves show the maximum, which correspond to the tip position. It means there exists negative thermal gradient ahead of the tip. In the early stage, the negative thermal gradient in liquid side is resulted from the initial undercooling. In one-dimensional calculation, the negative temperature gradient in front of interface disappears quickly and the growth of interface is

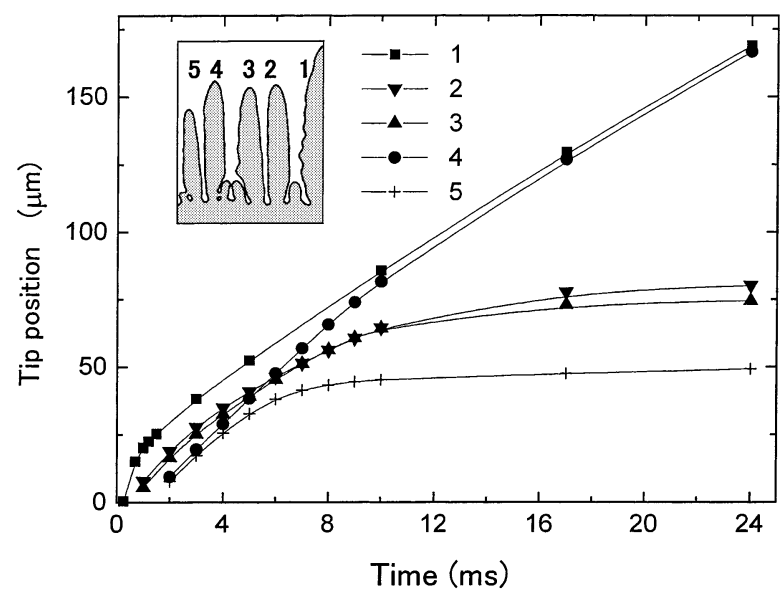

Fig. 3. The position of tips depending on time. The tip velocity of survived arm becomes almost constant and is estimated as $6 \mathrm{~mm} / \mathrm{s}$.

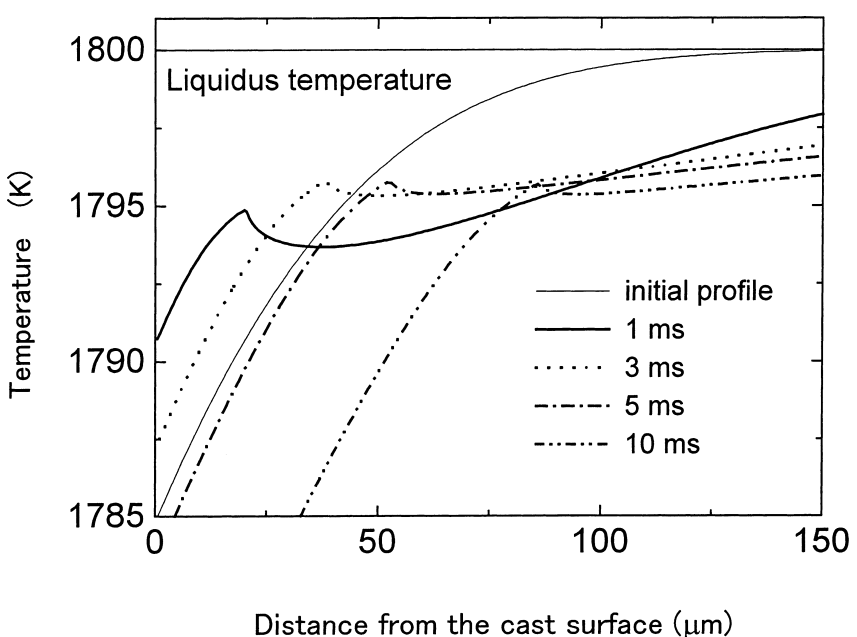

Fig. 4. Time change of temperature profile along to the leading arm. Negative thermal gradient is always observed both solid and liquid sides.

controlled by the heat extraction to the chill plate. On the other hand, since the reduced arms next to the leading arm grow slower or stop advancing in two-dimensional calculation, the amount of latent heat generation is smaller than that of leading tips. Hence the temperature of liquid phase in front of reduced arms is lower than that of a leading tip and the latent heat from the leading tip flows away through not only solid phase but also liquid phase (Fig. 5). These results show that the growth behavior of survived arms can be regarded as a directional free dendrite growth.

\subsection{Microstructure Change Depending on the Growth Direction and Phosphorus Addition}

Figures 6(a)-6(f) show the microstructure evolution when the angle between preferred growth orientation and heat flux direction is 30 degree. When the thermal Peclet number $\left(=\lambda_{1} V / \alpha\right)$, is small, the observed tilted angle is deviated from the prefered growth orientation toward the heat flux direction. ${ }^{20)}$ The tip velocity of $6 \mathrm{~mm} / \mathrm{s}$, however, is large enough and the primary and secondary arms grow along the preferred orientation in each case. Figures 7(a)7(d) show the microstructure change depending on the tilt- 
Fig. 5. Temperature distribution at $10 \mathrm{~ms}$. Contour line is drawn at every $0.2 \mathrm{~K}$. Since the liquid temperature ahead of stopped arm is lower than that of growing arm, the latent heat from leading tip flows into the side liquid.

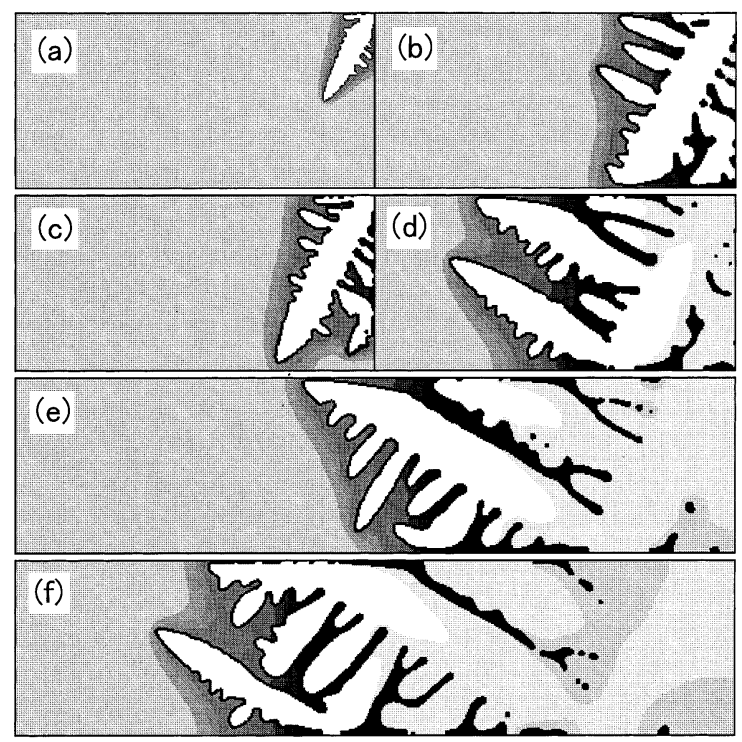

Fig. 6. The microstructure evolution when preferred growth orientation is tilted 30 degree. (a) $1.0 \mathrm{~ms}$, (b) $3.0 \mathrm{~ms}$, (c) $5.0 \mathrm{~ms}$, (d) $10 \mathrm{~ms}$, (e) $15 \mathrm{~ms}$, (f) $20 \mathrm{~ms}$.

ed angle between preferred growth orientation and the $x$ axis. The initial undercooling is $15 \mathrm{~K}$ and the tilted angle are set as $0,15,30$ and 45 degree, respectively. In case of zero degree, the secondary arms can grow on the both sides of a primary dendrite trunk. On the other hand, the secondary arms grow on one side in case of 15 and 30 degree because the solute diffusion is easily prevented by arm growing prior to it. In case of 45 degree, the secondary arm develops as large as the primary one because its growth condition is the same as the primary arm. In this way, the growth of secondary arm is sensitive to the growth direction of arms.

The microstructure change by phosphorus addition is analyzed. Figure 7(e) shows the microstructure of $\mathrm{Fe}-$ $0.5 \mathrm{~mol} \% \mathrm{C}-0.02 \mathrm{~mol} \% \mathrm{P}$ alloy. The primary and secondary arm spacings are reduced in the ternary alloy, and secondary arm spacing is 10 to $20 \%$ smaller than that in the binary alloy. Since the solute diffusivity of phosphorous is much smaller than that of carbon, it is easy to be segregated in the vicinity of the interface.

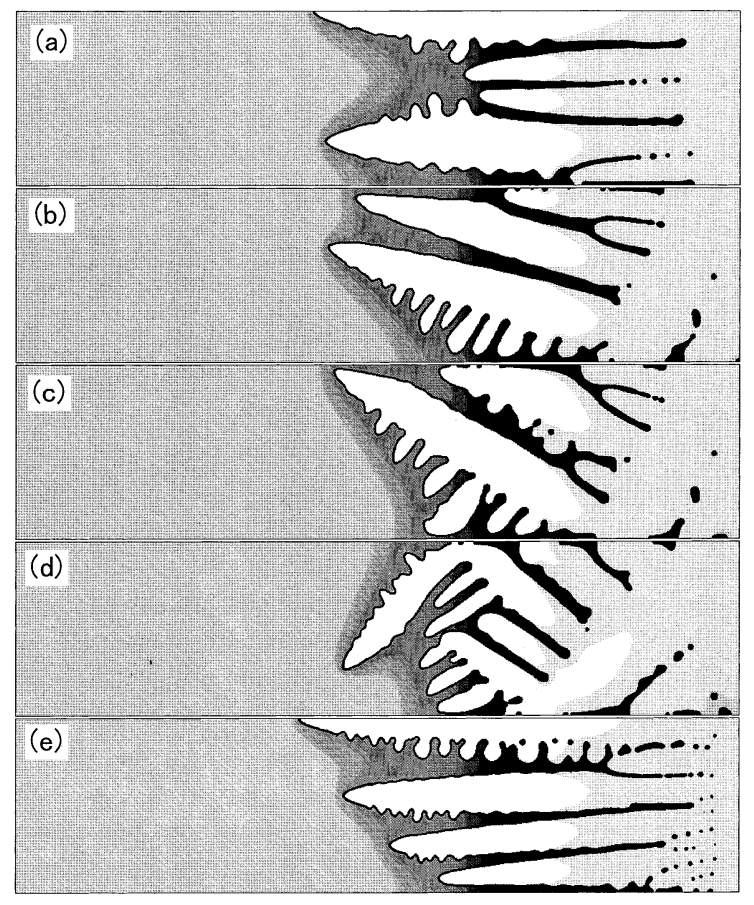

Fig. 7. Change of microstructure depending on tilted angle [(a)(d)] and the impurity (e). (a) 0 degree, (b) 15 degree, (c) 30 degree, (d) 45 degree, (e) Fe- $0.5 \mathrm{~mol} \% \mathrm{C}-0.02 \mathrm{~mol} \% \mathrm{P}$.

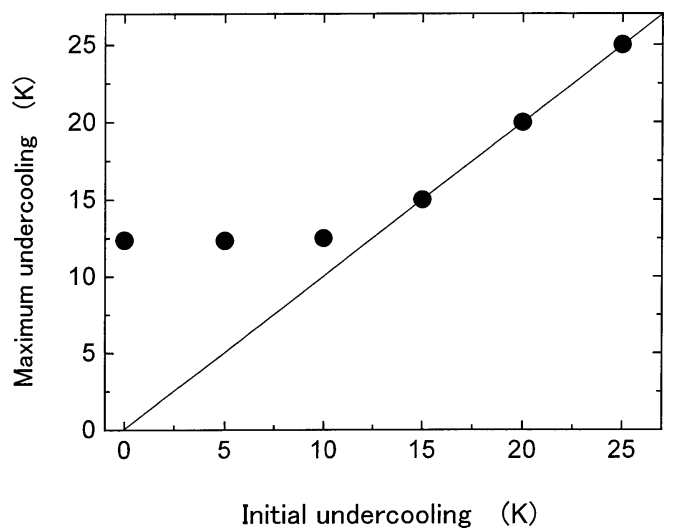

Fig. 8. Imposed initial undercooling vs. maximum undercooling. Even when the initial undercooling is zero, there is a certain undercooling independent of initial one.

\subsection{Temperature Change at the Melt Surface}

It is reported that the melt surface is largely undercooled before the solidification starts. ${ }^{3,6)}$ In the experiments, the surface temperature is measured as an average value in a certain area with a lot of nucleation points. Since the initial solid spacing represents a grain size, the averaged temperature of surface elements in the calculation area can be comparable to the experimentally measured one. Figure 8 shows the time change of the averaged surface temperature nucleated at 0,5 and $15 \mathrm{~K}$ undercooling, respectively. The shape of each curve is similar to that of the experiment. ${ }^{6}$ In case of no undercooling, the surface temperature decreases after the beginning of solidification because the solidification rate is very small and the temperature of the melt contacting the mold continues to decrease. The surface temperature begins to increase as the growth rate and the latent heat generation increases until the melt surface is covered 

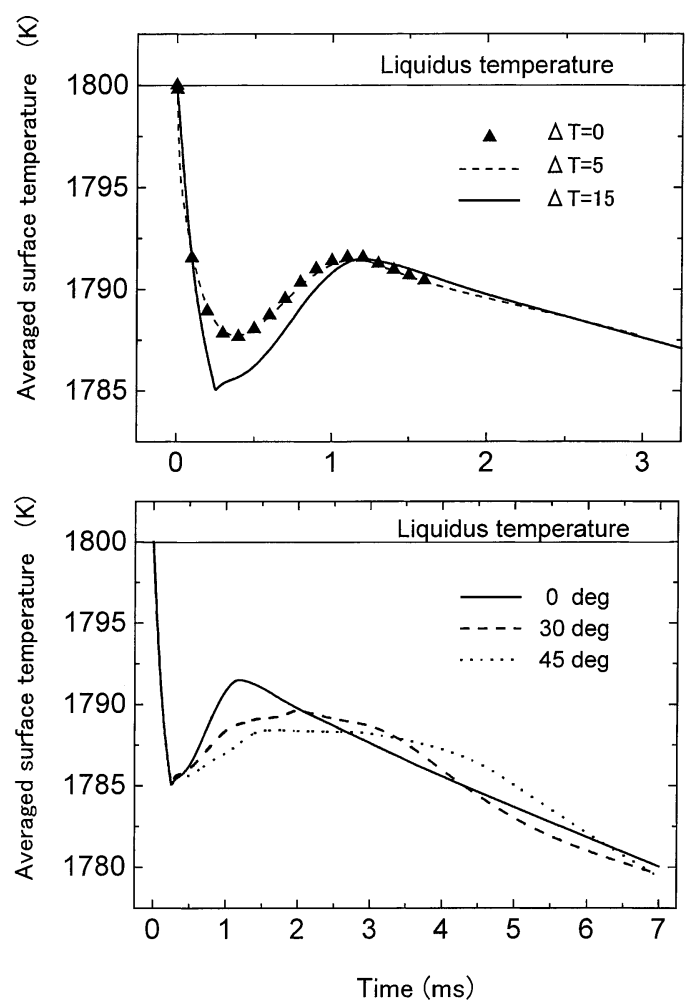

Fig. 9. The time change of averaged surface temperature depending on initial undercooling or tilted angle.

with solid phase. In case of initial undercooling of $5 \mathrm{~K}$, the shape of surface temperature curve is almost the same as that of no undercooling.

On the other hand, the temperature increases immediately just after the beginning of solidification when the initial undercooling is $15 \mathrm{~K}$. Since the initial undercooling is large enough, the latent heat generation resulting from the rapid solidification rate is large to increase the averaged surface temperature immediately. The relationship between initial undercooling and maximum undercooling of the averaged surface temperature is shown in Fig. 9. The maximum undercooling corresponds with the imposed one when the undecooling is larger than $15 \mathrm{~K}$. The surface temperature, however, is undercooled until a certain temperature even when the imposed undercooling is small. The microstructure evolution after nucleation, however, is not changed depending on the initial undercooling because the large undercooling is saturated with the latent heat generation at the very early stage of solidification.

Figure 9 shows that the change in surface temperature depends on the tilted angle. As shown in Fig. 6 there is a liquid phase between mold and initial leading arm in the early stage when preferred growth orientation is tilted. The liquid behaves as a heat sink at first due to its initial undercooling, and then a heat source when it solidifies. Therefore, the increase of surface temperature is more gradual than that of no tilted case. The change of surface temperature in an experimental sample will be averaged by these effects due to various growth directions. Hence the increase of the surface temperature in the experiment is more gradual than in the calculation. ${ }^{6}$

\section{Conclusion}

The microstructure evolution during the rapid solidification of $\mathrm{Fe}-\mathrm{C}$ and $\mathrm{Fe}-\mathrm{C}-\mathrm{P}$ alloys is simulated using the alloy phase-field model with thin interface limit parameters. The thermal field equation solved simultaneously and the double grid method and parallel computing technique are adopted to reduce the computational time. The transition of cellular/dendritic growth is observed after the competitive growth of cells when the leading tip advanced to about $100 \mu \mathrm{m}$ from the melt surface. The calculated primary and secondary arm spacing is in good agreement with experimental data which is estimated as $25 \mu \mathrm{m}$ and 10 to $5 \mu \mathrm{m}$, respectively. The velocity of a leading tip becomes constant and there is the negative thermal gradient not only on solid side but also ahead of the tip. From these results, the growth of a leading tip can be regarded as free unidirectional dendrite growth. The effects of the tilted angle and impurity on the microstructure are also studied. The calculated arm growth direction agrees with the preferred orientation and the secondary arms grow preferably towards inside of the melt when the growth direction is tilted. The small amount of phosphorus addition reduces both secondary and primary arm spacing since the partition coefficient and solute diffusivity of phosphorus are smaller than those of carbon. The result of time change in averaged surface temperature shows that the surface undercooling is always observed independent of nucleation temperature and the shape of the cooling curve becomes gentler when the growth direction is tilted from the direction of mean heat flux. The various factors affecting the microstructure are examined quantitatively and the wide potentiality of the phase-field model towards the rapid solidification problems has been demonstrated.

\section{Acknowledgement}

The authors are grateful to Prof. S.G. Kim of Kunsan National University and Prof. W. T. Kim of Chongu University for helpful discussion. The one of the author (M.O.) is financially supported by the Japan Society for the Promotion of Science (No. 05347).

\section{REFERENCES}

1) H. Mizukami, T. Suzuki and T. Umeda: Tetsu-to-Hagané, 10 (1991), 1672.

2) H. Mizukami, T. Suzuki and T. Umeda: Tetsu-to-Hagané, 5 (1992), 767.

3) T. Koseki and M. C. Flemings: Metall. Mater. Trans. A, 26A (1995), 2991.

4) K. Sakuma and T. Suzuki: Tetsu-to-Hagané, 81 (1995), 518.

5) T. Koseki and M. C. Flemings: Metall. Mater. Trans. A, 27A (1996), 3226.

6) H. Todoroki, R. Lertarom, A. W. Cramb and T. Suzuki: ISS Trans., (1999), 57.

7) T. Koseki and M. C. Flemings: ISIJ Int., 35 (1995), 611.

8) J. S. Langer: Directions in Condensed Matter, World Scientific, Singapore, (1986), 165.

9) A. A. Wheeler, W. J. Boettinger and G. B. McFadden: Phys. Rev. A, 45 (1992), 7424.

10) R. Kobayashi: Physica D, 63 (1993), 410.

11) S.-L. Wang, R. F. Sekerka, A. A. Wheeler, B. T. Murray, S. R. Coriell, R. J. Braun and G. B. Mcfadden: Physica D, 69 (1993), 189.

12) G. B. McFadden, A. A. Wheeler, R. J. Braun, S. R. Coriell and R. F. Sekerka: Phys. Rev. E, 48 (1993), 2016. 
ISIJ International, Vol. 42 (2002), No. 4

13) B. T. Murray, A. A. Wheeler and M. E. Glicksman: J. Cryst. Growth, 154 (1995), 386.

14) J. A. Warren and W. J. Boettinger: Acta Metall. Mater., 43 (1995), 689.

15) A. Karma and W.-J. Rappel: Phys. Rev. E, 53 (1996), R3017.

16) S. G. Kim, W. T. Kim and T. Suzuki: Phys. Rev. E, 60 (1999), 7186.
17) M. Ode, J. S. Lee, S. G. Kim W. T. Kim and T. Suzuki: ISIJ Int., 40 (2000), 870 .

18) S. Nishioka, Y. Tsuchida, T. Kitagawa, Y. Fujita, H. Sato and M. Ohtsuki: Trans. Iron Steel Inst. Jpn., 26 (1986), B147.

19) L.-T. Shiang and P. J. Wray: Metall. Trans. A, 20A (1989), 1191.

20) S. Akamatsu and T. Ihle: Phys. Rev. E, 56 (1997), 4479. 